\title{
St. Galler Paternoster und Credo
}

Sonderegger, Stefan ; Glaser, Elvira

DOI: https://doi.org/10.1515/9783110245509.1

Posted at the Zurich Open Repository and Archive, University of Zurich

ZORA URL: https://doi.org/10.5167/uzh-78219

Book Section

Published Version

Originally published at:

Sonderegger, Stefan; Glaser, Elvira (2013). St. Galler Paternoster und Credo. In: Bergmann, Rolf. Althochdeutsche und altsächsische Literatur. Berlin, Germany; Boston, USA: de Gruyter, 100-103.

DOI: https://doi.org/10.1515/9783110245509.1 
Verfasser-Datenbank

Autoren der deutschsprachigen Literatur und des deutschsprachigen Raums: Von den Anfängen bis zur Gegenwart

Version 2 (Erschienen 17.09.2014). Andere Version ansehen:

Verfasserlexikon - Die deutsche Literatur des Mittelalters

De Gruyter (Berlin, Boston) 2014

10.1515/vdbo.vima.1211 v2

Online erschienen 2014

\section{'St. Galler Paternoster und Credo'}

Stefan Sonderegger, Elvira Glaser

Epoche: Mittelalter (bis 1500)

Aufzeichnungsdatum: 8. Jh. (Ende)

Werkart: Gebet

Trotz ihrer inhaltlich verschiedenen und zum Teil auch nach dem althochdeutschen Sprachstand differenzierbaren Teile (Abrogans Ka, Kb; althochdeutscher Anhang mit Paternoster und Credo) stellt die Handschrift eine relative Einheit in paläographischer und kodikologischer Hinsicht dar, deren letzte beiden Lagen S. 291-323 nicht erst später, sondern „mit einem kaum ins Gewicht fallenden zeitlichen Abstand“ (B. Bischoff) hinzugefügt worden sind. Mit spekulativer Argumentation verficht G. Must 1981 eine Abschrift von Paternoster und Credo um 760 womöglich in St. Gallen. St. Müller (Althochdeutsche Literatur, S. 349) geht, wie andere vorher, von einem ursprünglich selbstständigen Teil aus. Nach B. Bischoff sind 'St. Galler Paternoster und Credo' wie der ahd. $\rightarrow$ 'Abrogans' derselben Handschrift in einem uns unbekannten klösterlichen Scriptorium des deutschsprachigen Südwestens in den letzten Jahren des 8. Jahrhunderts niedergeschrieben worden. Der Wortschatz der kurzen Textstücke (47 und 82 Wörter) ist bei E. Seebold (Chronologisches Wörterbuch [I]) als Überlieferung des 8. Jahrhunderts erfasst. Die Handschrift muss bereits in dieser Zusammensetzung in die Klosterbibliothek (heute Stiftsbibliothek) St. Gallen gelangt sein, was vielleicht schon in der 1. Hälfte des 9 . Jahrhunderts 'Libri glosarum volumina VIII' des ältesten Bücherverzeichnisses aus der Zeit nach 850 verbirgt (J. Duft). Der Name 'St. Galler Paternoster und Credo' ist also überlieferungsgeschichtlich und nicht entstehungsgeschichtlich begründet, und es darf nicht, wie früher üblich, von einem St. Galler Übersetzer gesprochen werden. Die Sprache von 'Paternoster' und 'Credo' lässt sich gegenüber dem 'Abrogans'-Sprachstand derselben Handschrift (Ka, Kb) als jüngeres Frühalthochdeutsch alemannischer Prägung bestimmen, wobei die alemannischen Kernmerkmale teils weniger deutlich (so gegenüber Ka), teils deutlicher (so gegenüber Kb) sind als im St. Galler 'Abrogans' (St. Sonderegger, 1977). Ob eine Abschrift aus älterer Vorlage wie im 'Abrogans'-Teil der Handschrift vorliegt, ist trotz einiger Fehler und Verbesserungen nicht sicher auszumachen. Die beiden Texte gehen aber wohl auf denselben Übersetzer zurück. A. Greule wirft jüngst eine Regensburger Entstehung der St. Galler Paternosterübersetzung auf. Die Platzierung der beiden Texte am Schluss der Handschrift gibt dem Codex eine geradezu geistlich-liturgische Abrundung.

\section{Einordnung}

Das nach Übersetzungsweise und Sprachstand archaische Denkmal aus den letzten Jahren des 8. Jahrhunderts eröffnet als älteste diesbezügliche Fassung die verhältnismäßig reiche, an verschiedenen Überlieferungsorten unabhängig voneinander entstandene althochdeutsche Paternoster- und Credo-Übertragung, welche von der frühalthochdeutschen Zeit (u. a. $\rightarrow$ 'Weißenburger Katechismus', $\rightarrow$ 'Freisinger Paternoster') über die normalalthochdeutsche Tatianübersetzung ( $\rightarrow$ 'Tatian') (Paternoster) bis zu $\rightarrow$ Notker III. von St. Gallen (Oratio dominica und Symbolum apostolorum) reicht und deren schriftliche Fixierung offensichtlich durch Karls d. Gr. 'Admonitio generalis' vom 23. März 789 ausgelöst worden ist. Dabei bleibt die Möglichkeit noch älterer volkssprachiger Übersetzungen im liturgischen Rahmen schon des 8. Jahrhunderts (G. Must zu Paternoster und Credo) grundsätzlich bestehen, wenn auch entsprechende Stützen dafür nur indirekt über die Textphilologie von Übersetzung und dahinter stehender lateinischen Grundfassung erbracht werden können: dennoch dürfen die vorliegenden althochdeutschen Texte des Codex Sang. 911 nach Sprachstand und Überlieferung vorderhand nicht weiter zurück datiert werden (anders wiederholt G. Must, Anfang des 8. Jahrhunderts), mag man in ihnen auch stellenweise Formulierungen einer älteren liturgischen Tradition erblicken. Auch aus der Textform des Credo schließt G. Must auf westgallisch-irische Zusammenhänge. Hinsichtlich einiger Wortschatzelemente wird das 'Paternoster' jüngst wieder von A. Greule (2012) in den Kontext der gotisch-arianischen Mission gestellt. Umstritten ist in der Forschung auch nach wie vor die Deutung einzelner Merkwürdigkeiten im Übersetzungsvorgang, der als solcher eine interlinearartige Struktur mit nahezu vollständiger Wort-für-Wort- oder Form-für Form-Entsprechung vom Lateinischen zum Althochdeutschen aufweist (St. Sonderegger, 1965, anders G. Must 1981): vor allem die Stellen ahd. uuihi namun dinan (für lateinisch sanctificetur nomen tuum), althochdeutsch uns sculdikem (für lateinisch debitoribus nostris), althochdeutsch kisca[f]t himiles enti erda (für lateinisch creatorem caeli et terrae), althochdeutsch kimartrot in kiuualtiu pilates (für lateinisch passus sub Pontio Pilato) und althochdeutsch urlaz suntikero (für lateinisch remissionem peccatorum). Gegen die traditionelle Deutung als Übersetzungsfehler (z. B. A. Masser, H. Eggers sowie tendenziell auch W. Haubrichs) hat W. Betz $(1961,1964,1965)$ darin einen karolingischen erklärenden Übertragungsstil sehen wollen, welchen er zum Teil in der exegetischen Kommentarliteratur der Zeit verankern kann (vgl. zuletzt ähnlich auch A. Greule 2012 [2004]). Näher bei W. Betz steht auch G. Must, welcher hinter der packend-einfachen althochdeutschen Diktion des Paternoster die charakteristischen Züge der Liturgie des wandernden Mönchtums, insbesondere der irischen Mission in Süddeutschland, erkennen will und im Credo sprechsprachliche Züge neben der Tradierung alter Textvarianten sieht. Man wird vermittelnd alle drei Möglichkeiten je alternierend für die verschiedenen schwierigen oder vom vergleichbaren althochdeutschen Übersetzungsstand abweichenden Stellen bedenken müssen, Kenntnis des genauen lateinischen Wortlauts der Vorlagen abhängt (Paternoster: Vulgata, Vetus Latina, liturgische Abweichungen, bekannte oder benutzte Kommentare; Credo nach dem sog. Textus receptus der westlichen Kirche im Frühmittelalter, der als forma recentior eine erweiterte Tochterform der forma vetustior des Apostolischen Glaubensbekenntnisses ist). Zur Grundlage des Credo äußert sich auch G. Must (1981). E. Meineke hat jüngst das Paternoster als Beispiel eines speziellen für pastorale Texte geeigneten „Übersetzungsstils“ mit starker 
„syntaktischer Anlehnung an die autoritative lateinische Vorlage“ bewertet. Bei R. Hochholzer wird die Wortwahl (für 'Himmel' und 'Hölle') der beiden frühen pastoralen Texte als Vorbild für die entstehende deutsche Literatur bewertet. H. Tiefenbach sieht die beiden Texte als Beispiele für die „Bewältigung der sprachlichen Vermittlungsaufgabe" zu Beginn der althochdeutschen Schriftlichkeit und plädiert für eine differenzierte Sicht auf die Übersetzungsleistung. W. Haubrichs stellt das 'St. Galler Paternoster und Credo' als „nicht ganz fehlerfreie Interlinearversionen“ in den Kontext „schulische[r] Bearbeitung“.

\section{Überlieferung}

St. Gallen, Stiftsbibl., Cod. 911 renthält als abschließenden dritten Teil S. 320 (recte 319, die Seitenzahl 303 wurde beim Paginieren übersprungen) bis S. 322 (recte 321 ) auf schadhaftem Pergament die frühalthochdeutsche Übersetzung von Paternoster (S. 320) und Credo (S. 321-322) ohne den entsprechenden lateinischen Text, wobei das Paternoster S. 319 (recte 318) unten mit einem Kreuz und dem abgekürzten latateinischen Pater noster eingeleitet wird, während das Glaubensbekenntnis S. 321 oben mit teilweise unzialem CREDO IN DIEU]M überschrieben ist. Der erste Teil der Handschrift (S. 4-289) enthält das lateinisch-althochdeutsche Abrogans-Glossar ( $\rightarrow$ 'Abrogans deutsch'), der zweite Teil (S. 292-319, recte 318) Kap. 1-17 des Liber ecclesiasticorum dogmatum von Gennadius von Marseille.

Abbildungen (Auswahl):

H. Fischer, Schrifttafeln zum althochdeutschen Lesebuch, Tübingen 1966, Tafel 2.

B. Bischoff - J. Duft - St. Sonderegger, Das älteste deutsche Buch. Die Abrogans-Handschrift der Stiftsbibliothek St. Gallen. Im Faksimile hg. u. beschrieben, St. Gallen 1977.

St. Sonderegger, Althochdeutsche Sprache und Literatur. Eine Einführung in das älteste Deutsch. Darstellung und Grammatik, 3.A., Berlin/New York 2003, S. 94 , 96, 98.

Faksimile des Paternoster in Cimelia Sangallensia. Hundert Kostbarkeiten aus der Stiftsbibliothek St. Gallen. Beschrieben von K. Schmuki - P. Ochsenbein - C. Dora, St. Gallen 1998, S. 33.

Digitalisat $\nearrow$

\section{Ausgaben}

Editio princeps: M. Freher, Orationis dominicae et symboli apostolici Alamannica versio vetustissima, Heidelberg 1609.

Weitere Ausgaben (Auswahl), die die Texte unterschiedlich und teilweise unter Hinzufügung von Interpunktion sowie mit Anmerkungen präsentieren:

H. Hattemer, Denkmahle des Mittelalters. St. Gallen's altteutsche Sprachschätze. Gesammelt und herausgegeben, I-III, St. Gallen [1844-1847], Nachdruck Graz 1970, I, S. $324 f$.

K. Müllenhoff - W. Scherer, Denkmäler deutscher Poesie und Prosa aus dem VIII-XII Jahrhundert, 3. A. von E. Steinmeyer, Berlin 1892, Nachdruck 1964, Nr. LVII, I, S. 209, II, S. 342-344.

E. von Steinmeyer, Die kleineren althochdeutschen Sprachdenkmäler, Berlin 1916, Nr. V, S. 27 (hier S. 27f. über ältere Editionen, dazu auch J. Duft und B. Hertenstein).

W. Braune, Althochdeutsches Lesebuch, 17. A. bearb. v. E. A. Ebbinghaus, Tübingen 1994 (1. A. 1875), S. 11-12 (Nr. VI).

H. Fischer, Schrifttafeln zum althochdeutschen Lesebuch, Tübingen 1966, S. $5^{\star}$.

B. Bischoff - J. Duft - St. Sonderegger, Das älteste deutsche Buch, S. 306-307 (Transkription des ahd. Anhangs v. St. Sonderegger).

St. Müller, Althochdeutsche Literatur. Eine kommentierte Anthologie, Althochdeutsch / Neuhochdeutsch, Stuttgart 2007, S. 172, 174 (Paternoster), 182 (Credo) (mit Übersetzung).

\section{Literatur}

Ältere Literatur bei K. Müllenhoff - W. Scherer, Denkmäler deutscher Poesie und Prosa aus dem VIII-XII Jahrhundert, 3. A. von E. Steinmeyer, Berlin 1892, Nachdruck 1964, II, S. 342-344.

Google Scholar

E. von Steinmeyer, Die kleineren althochdeutschen Sprachdenkmäler, Berlin 1916, S. 27 f. Google Scholar

G. Ehrismann, Geschichte der deutschen Literatur bis zum Ausgang des Mittelalters, I. Die althochdeutsche Literatur, 2. A. München 1932 , S. 306. Google Scholar

W. Achnitz (Hg.), Das Geistliche Schrifttum von den Anfängen bis zum Beginn des 14. Jahrhunderts, Berlin/Boston 2011, S. 12-14 (Literaturzusammenstellung). Google Scholar

G. Baesecke, Unerledigte Vorfragen der ahd. Textkritik und Literaturgeschichte. I. Die Vaterunser vor Notker, Beiträge zur Geschichte der deutschen Sprache und Literatur 69 (1947) S. 361-365.

Google Scholar

W. Betz, Zum St. Galler Paternoster, Beiträge zur Geschichte der deutschen Sprache und Literatur 82 (Halle 1961) Sonderband. Festschrift Elisabeth Karg-

Gasterstädt, S. 153-156.

Google Scholar

W. Betz, Zum St. Galler Credo, in: Festschrift für Taylor Starck, The Hague 1964, S. 102-105.

Google Scholar

W. Betz, Ahd. kiscaft 'creator', Münchener Studien zur Sprachwissenschaft 18 (1965) S. 5-11. Google Scholar

W. Betz, Karl der Große und die Lingua Theodisca, in: Karl der Große, Lebenswerk und Nachleben, II. Das geistige Leben, hg.. v. B. Bischoff, 1965, S. 300-306 (hier über beide Denkm.).

Google Scholar 
B. Bischoff - J. Duft - St. Sonderegger, Das älteste deutsche Buch, S. 83-137.

Google Scholar

H. Brauer, Die Bücherei von St. Gallen und das althochdeutsche Schrifttum, Hermaea XVII, 1926, S. 52, 87. Google Scholar

H. Eggers, Deutsche Sprachgeschichte, I, Reinbek bei Hamburg 1963, S. $194 f$. Google Scholar

A. Greule, Über den Erkenntniswert der Etymologie religiöser Begriffe: nhd. weih. 1. St. Galler Paternoster, in: Sakralität. Studien zu Sprachkultur und religiöser Sprache, Tübingen 2012, S. 39-44.

Google Scholar

A. Greule, Über die Anfänge deutscher Sprachkultur und Sprachkultivierung, in: Sakralität. Studien zu Sprachkultur und religiöser Sprache, Tübingen 2012, S. 3-17, S.

10f. (zuerst in: Entstehung des Deutschen. Festschrift Heinrich Tiefenbach 2004, S. 133-142). Google Scholar

W. Haubrichs, Die Anfänge. Versuche volkssprachiger Schriftlichkeit im frühen Mittelalter (ca. 700-1050/60), Geschichte der deutschen Literatur von den Anfängen bis zum Beginn der Neuzeit. Hg. v. J. Heinzle, I. Von den Anfängen zum hohen Mittelalter. Teil 1, 2. Auflage, Tübingen 1995, S. 237. Google Scholar

B. Hertenstein, Joachim von Watt (Vadianus), Bartholomäus Schobinger, Melchior Goldast. Die Beschäftigung mit dem Althochdeutschen von St. Gallen in Humanismus und Frühbarock, Das Althochdeutsche von St. Gallen. Texte und Untersuchungen zur sprachlichen Überlieferung St. Gallens vom 8 . bis zum 12. Jahrhundert 3, Berlin/New York 1975, S. 130, 132f., 188, 189a.

Google Scholar

R. Hochholzer, Himmel und Hölle. Onomasiologische und semasiologische Studien zu den Jenseitsbezeichnungen im Althochdeutschen, Frankfurt u.a. 1996, S. 114119. Google Scholar

A. Masser, Die althochdeutschen Übersetzungen des Vaterunsers, Beiträge zur Geschichte der deutschen Sprache und Literatur 85 ( Tüb. 1963 ) S. $35-45$. Google Scholar

E. Meineke, Textgebundene Formen der deutsch-lateinischen Zweisprachigkeit im frühen Mittelalter, in: M. Baldzuhn - Ch. Putzo, Mehrsprachigkeit im Mittelalter,

Berlin 2011, S. 109-146, zu St. G. Paternoster S. 110-113, 144.

Google Scholar

St. Müller, Althochdeutsche Literatur. Eine kommentierte Anthologie, Althochdeutsch / Neuhochdeutsch, Stuttgart 2007, S. 349-350, 353-354.

Google Scholar

G. Must, Das St. Galler Paternoster, in: Akten d. V. Internat. Germanisten-Kongresses Cambridge 1975, Jahrbuch für Internationale Germanistik, Reihe A,

Kongreßberichte, II, 1976, S. 396-403.

Google Scholar

G. Must, Das altalemannische Wort 'kiscat', in: Akten des VI. Internationalen Germanisten-Kongresses Basel 1980, II, Bern 1980, S. $399-403$. Google Scholar

G. Must, Das St. Galler Credo, Frühmittelalterliche Studien 15 (1981) S. 371-386. Google Scholar

St. Sonderegger, Frühe Übersetzungsschichten im Althochdeutschen, in: Philologia Deutsch, Festschrift Walter Henzen, Bern 1965 , S. 111. Google Scholar

St. Sonderegger, Althochdeutsch in St. Gallen, Ergebnisse und Probleme der althochdeutschen Sprachüberlieferung in St. Gallen vom 8. bis ins 12. Jahrhundert, Sigmaringen 1970, S. 57-64.

Google Scholar

St. Sonderegger, Althochdeutsche Sprache und Literatur. Eine Einführung in das älteste Deutsch. Darstellung und Grammatik, 3. Auflage, Berlin/New York 2003, S. 91-96

Google Scholar

H. Tiefenbach, Zur sprachlichen Christianisierung im frühen Deutschen, in: O doskonałości. Materiały z konferencji 21-23 maja 2001r., I, Łódz. 2002, S. 341-366. Google Scholar

St. Sonderegger - E. Glaser, 'St. Galler Paternoster und Credo'; in: R. Bergmann, Althochdeutsche und altsächsische Literatur, Berlin/Boston 2013, S.100-103 (= Grundlage des vorliegenden Artikels).

Google Scholar

\section{Schlagworte}

\section{Personen}

Gennadius v. Marseille (Massiliensis)

Karl I. der Große, K., Kg. d. Franken

Notker III. v. St. Gallen (N. Labeo, N. Teutonicus, d. Deutsche) OSB

\section{Handschriften}

St. Gallen, Stiftsbibl., Cod. 91 
Zugang bereitgestellt von: UZH Hauptbibliothek / Zentralbibliothek Zürich

Copyright $\odot$ 2011-2017 by Walter de Gruyter GmbH

Powered by PubFactory 